Article

\title{
SINR-Based MCS Level Adaptation in CSMA/CA Wireless Networks to Embrace IoT Devices
}

\author{
Soohyun Cho \\ Department of General Studies, Hongik University, Seoul 04066, Korea; cho.soohyun@hongik.ac.kr; \\ Tel.: +82-2-320-3350
}

Received: 20 September 2017; Accepted: 16 October 2017; Published: 19 October 2017

\begin{abstract}
This paper presents an automatic modulation and coding scheme (MCS) level adaptation algorithm to embrace Internet of Things (IoT) devices by improving the area spectral efficiency of carrier-sense multiple access with collision avoidance (CSMA/CA) wireless networks. In the proposed algorithm, senders of CSMA/CA wireless networks use the signal to interference plus noise ratio of acknowledgment frames from their receivers to estimate channel statuses between the senders and the receivers. Using the estimated channel status of each receiver, senders control sending rates of traffic by adjusting MCS levels of packets destined for each receiver. We use Poisson point processes (PPPs) to model the locations of participating nodes (i.e., access points and wireless devices) in a given area. We evaluate the effectiveness of the proposed algorithm using an event-driven ns-2 simulator for various PPP densities of access points and wireless devices.
\end{abstract}

Keywords: Internet of Things; modulation and coding scheme; rate adaptation; carrier-sense multiple access with collision avoidance (CSMA/CA); IEEE 802.11; Poisson point processes

\section{Introduction}

Owing to recent advances in Internet of Things (IoT) [1] technologies, it is expected that emerging IoT-based services will enhance the quality of our daily lives significantly in many areas. Some examples are smart homes [2], smart vehicles [3], and smart safety [4]. However, for widespread deployment of IoT-based services, IoT technologies still need to be improved in many aspects such as power [5], connectivity, and privacy [6]. For connectivity, it is expected that IoT devices will use various kinds of wireless networks. These wireless networks can be operator-managed, wide-area wireless networks such as 3G, 4G, and 5G cellular networks [7] but also can be unmanaged, license-free carrier-sense multiple access with collision avoidance (CSMA/CA) based wireless networks such as IEEE 802.11 [8] and IEEE 802.15.4 [9].

In this paper, we focus on the use of IEEE 802.11 wireless networks for the connectivity of IoT devices because IoT devices may benefit from using license-free wireless networks, especially IEEE 802.11, owing to the high penetration of IEEE 802.11 wireless networks in many areas including homes and offices. However, to accommodate the expected high density of the IoT devices using CSMA/CA-based IEEE 802.11 wireless networks along with existing wireless devices (i.e., smartphones and laptops), participating devices need to efficiently utilize network resources such as frequencies and spaces. The area spectral efficiency of uncoordinated wireless networks can be captured with metrics such as the transmission capacity [10], derived using stochastic geometry, and point process theories [11].

In wireless communications, the signal to interference plus noise ratio (SINR) is a key metric representing the wireless channel status, which affects the bit error rates of the packets [12]. However, it is hard to accurately estimate the ever-changing status of wireless channels especially when multiple senders and receivers are trying to communicate at the same time using a sophisticated 
collision avoidance mechanism such as CSMA/CA. In this paper, we suggest leveraging the SINRs of acknowledgment (ACK) frames in CSMA/CA wireless networks to estimate the channel statuses between senders and receivers since most license-free wireless networks such as IEEE 802.11 and IEEE 802.15.4 adopt half-duplexing to more efficiently utilize frequency bands (i.e., both downlink and uplink traffic use the same frequency band). Here, we are assuming that there are high correlations between downlink channels for data packets and uplink channels for ACK frames on short time scales. The contributions of this paper can be summarized as follows:

- Using the SINRs of ACK frames, we propose a novel modulation and coding scheme (MCS) level adaptation algorithm: senders adapt data sending rates by adjusting MCS levels of packets to be sent to their receivers based on the estimated channel statuses from the SINRs of ACK frames. In addition, senders decrease the MCS levels of packets in the absence of ACK frames after a given number of retransmissions (i.e., a retry limit event occurs).

- We demonstrate and evaluate the performance of the proposed algorithm using the ns-2 [13] network simulator (version 2.35) for IEEE 802.11a [14] wireless networks with various numbers of participating nodes (i.e., access points and wireless devices) distributed in a given area using independent homogeneous Poisson point processes (PPPs).

The remainder of this paper is organized as follows: in Section 2, we survey related work on the research for adapting data sending rates in CSMA/CA wireless networks. In Section 3, we explain the proposed algorithm in detail with examples and pseudo code. In Section 4, we demonstrate the behavior of the proposed algorithm in both single cell and multi-cell environments using simulations. In Section 5, we evaluate the performance of the proposed algorithm with various PPP densities of access points and users. Section 6 concludes this paper.

\section{Related Work}

Considerable research has been conducted on adapting data sending rates in IEEE 802.11 wireless networks. In this section, we briefly introduce some of the results and other related research.

In [15], the authors propose adapting data sending rates based on request-to-send/clear-to-send (RTS/CTS) messages to differentiate frame collisions from frame transmission failures. In [16], the authors suggest making receivers estimate the channel status and return the results by embedding them in ACK frames. In [12], the authors use probing packets to estimate the channel status. In [17], the authors propose using bit error rates to control data sending rates. In [18], the authors suggest using the error vector magnitude (EVM) of orthogonal frequency division multiplexing (OFDM) symbols to control data sending rates. In [19], the authors use group request-to-send/group clear-to-send (G-RTS/G-CTS) messages to improve overall network throughput in wireless mesh networks. The proposed algorithm in this paper is different from these approaches mainly because senders adapt their sending rates using ACK frames without requiring any modifications to the existing CSMA/CA mechanism.

Recently, researchers have been using theories from stochastic geometry to analyze various kinds of issues related to wireless networks, such as SINR distributions and outage (or coverage) probabilities for a given target SINR [20] in cellular networks and the transmission capacity [21] for wireless networks that use simple medium access control (MAC) protocols such as slotted ALOHA [22]. Compared to cellular networks and wireless networks of simple MAC protocols, analyzing IEEE 802.11 wireless networks seems more challenging owing to the dynamic nature of the CSMA/CA mechanism adopted in IEEE 802.11 standards. In [23], the authors analyze CSMA/CA wireless networks with stochastic geometry and show coverage probabilities with different PPP densities of nodes. In [24], the authors analyze interference in CSMA/CA wireless networks. In [25], the authors show distributions of throughputs with different PPP densities of nodes.

The proposed algorithm in this paper heavily depends on the accuracy in the measurement of the SINRs. For accurate measurement of the SINRs of the ACK frames, we use the IEEE 802.11Ext module 
from [26] in ns-2 simulations. Within the module, nodes not only use signal strengths and arrival times of packets but also use the sizes and transmission speeds of the arrived packets (i.e., durations) to calculate cumulative power at the nodes. The cumulative power is used to calculate the SINRs of packets when and while they are received. Figure 1 illustrates the changes of the cumulative power (described as cumulative noise in the figure) when packets of different received signal strengths and durations arrive at a node [27].

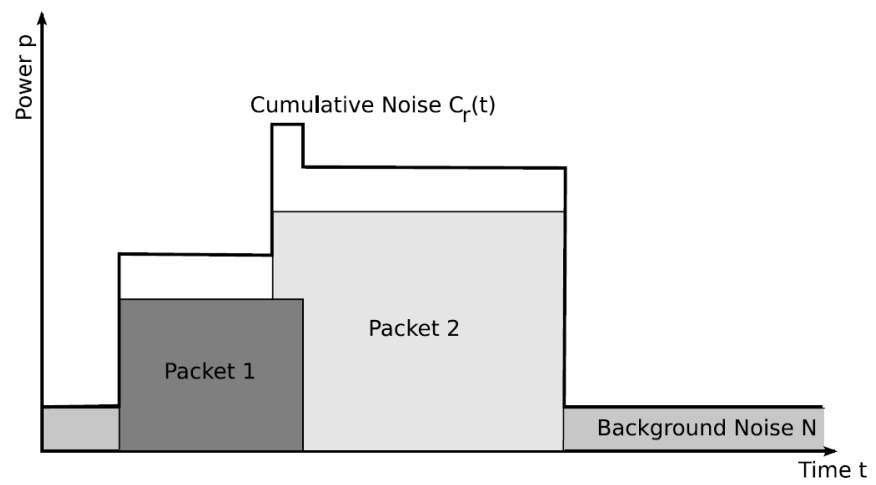

Figure 1. Changes of cumulative power (Figure 5.1 in [27]/CC BY).

\section{Proposed Algorithm}

\subsection{SINR and MCS}

We consider wireless networks consisting of $N$ nodes in a two-dimensional Euclidean space. For the calculation of the signal strengths and SINRs of received packets, we use a simple radio transmission model similar to the one used in [28] (Equation (1)) except for the random channel gains for simplicity in analysis: We assume that a node of interest is located at $x_{0}$ without loss of generality, and calculates the signal strength $\left(P_{i}\right)$ of a received packet sent from node $i$ located at $x_{i}$ as

$$
P_{i}=A \cdot P_{t}\left\|x_{0}-x_{i}\right\|^{-\alpha},
$$

where $i=1,2,3, \ldots, N-1, A$ is a constant to represent radio environments such as antenna gains, $P_{t}$ is the transmit power of nodes, $\alpha$ is the path loss exponent parameter, and $\|\cdot\|$ represents the Euclidian norm (When the distance between a sender and a receiver is less than 1 meter, the Euclidian norm is set to 1 to prevent possible overestimation of the received signal strengths from the arbitrary proximity of nodes owing to the property of PPP). In this paper, $A$ is set to $\sim 5.06, P_{t}$ is set to $100 \mathrm{~mW}, \alpha$ is set to 4 , and we do not consider short-term fading effects such as shadowing.

By using Equation (1) and the definition of SINR, when the node at $x_{0}$ is communicating with node $i$, the SINR of a received packet from node $i, \operatorname{SINR}_{i}$, is calculated as

$$
\operatorname{SINR}_{i}=\frac{P_{i}}{\sum_{j \neq i} A \cdot P_{t}\left\|x_{0}-x_{j}\right\|^{-\alpha} \mathbb{1}_{\{j \text { interferes with } i\}}+W^{\prime}}
$$

where $x_{j}$ is for locations of nodes whose packets interfere with desired packets from node $i$, and $\mathbb{1}$ is the indicator function. Thus, $\sum_{j \neq i} A \cdot P_{t}\left\|x_{0}-x_{j}\right\|^{-\alpha} \mathbb{1}_{\{j \text { interferes with } i\}}$ represents the cumulative power (i.e., interference) of interfering packets while the desired packet from node $i$ is being received. $W$ is the thermal noise power at receivers and it is assumed to be $-126 \mathrm{dBW}$ in this paper.

In IEEE 802.11 standards, such as IEEE 802.11a, multiple MCS levels are defined [14]. Each MCS level requires satisfaction of the minimum SINR for correct demodulation of received packets of the MCS level. For example, the IEEE 802.11Ext module in ns-2, which we use for the evaluation of the proposed algorithm, supports four MCS levels [29], as listed in Table 1 (Note that ACK frames are also 
sent using the MCS levels). In Table 1, we also list the (physical layer) transmission speed and the minimum SINR requirement $\left(\tau_{k}\right)$ of each MCS level $(k)$.

Table 1. MCS levels supported by the IEEE 802.11Ext module.

\begin{tabular}{cccc}
\hline MCS Level $(k)$ & MCS & Transmission Speed (Mbps) & Min. SINR $\left(\tau_{k}\right)(\mathrm{dB})$ \\
\hline 0 & BPSK, 1/2 coding & 6 & 5 \\
1 & QPSK, 1/2 coding & 12 & 8 \\
2 & 16QAM, 1/2 coding & 24 & 15 \\
3 & 64QAM, 3/4 coding & 54 & 25 \\
\hline
\end{tabular}

\subsection{Automatic-MCS-Level Adaptation}

In CSMA/CA wireless networks, when a node receives data packets successfully, the node sends back ACK frames to the sender of the data packets. In the proposed algorithm in this paper, a sender uses the SINRs of the ACK frames from its receiver to estimate the channel status between the sender and the receiver and adjusts the MCS levels of subsequent packets to be sent to the receiver. However, to avoid frequent updates of MCS levels caused by possible abrupt and frequent changes in the measured SINRs, we use (exponentially weighted) moving averaged SINR values $\left(\operatorname{avg} S I N R_{i}\right)$ instead of the instantaneous SINR value $\left(S I N R_{i}\right)$ of each ACK frame from receiver $i$.

As a result, $\operatorname{avg} \operatorname{SINR} R_{i}$ is calculated with every new $\operatorname{SINR}_{i}$ as

$$
\operatorname{avgSINR} i=\alpha \cdot \operatorname{avgSINR} R_{i}+(1-\alpha) \cdot \operatorname{SINR}_{i}
$$

where $\alpha$ is the smoothing factor, which is set to 0.9 in this paper. The value is set similarly to that used to calculate the moving averaged round-trip times of packets in transmission control protocol (TCP) [30] because both TCP and the proposed algorithm work in a similar time scale although they are in different communication layers.

In the proposed algorithm, a sender starts sending traffic to its receiver $i$ with the lowest MCS level (e.g., 0 in Table 1). Whenever an ACK frame from receiver $i$ arrives, the MCS level for receiver $i$ is increased by one if $\operatorname{avg} S I N R_{i}$ for receiver $i$ is higher than the minimum SINR requirement of the next higher MCS level unless it is the highest level (e.g., 3 in Table 1). If $a v g S I N R_{i}$ is less than the minimum SINR requirement of the current MCS level, the MCS level is decreased by one unless it is the lowest MCS level. Otherwise, the MCS level for receiver $i$ remains unchanged.

In addition, if the sender detects a retry limit event (A retry limit event occurs when the number of consecutive ACK timeouts of a data packet reaches a limit. By default, the long retry limit is 4 and the short retry (i.e., RTS/CTS is not used) limit is 7 [8].), the MCS level is decreased by one unless it is the lowest one. This decrease is necessary because the retry limit event may have occurred as a result of an MCS level that the receiver could not decode. Furthermore, $a v g S I N R_{i}$ is set to the middle value of the new minimum SINR requirement and the previous minimum SINR requirement to avoid additional timeouts that may occur while updating the moving averaged SINRs.

The pseudo code in Algorithm 1 summarizes the proposed algorithm using the MCS levels in Table 1 as an example (Note that the proposed algorithm does not control MCS levels of ACK frames. The MCS levels of ACK frames are always set to the lowest one (i.e., 0$)$ ). 


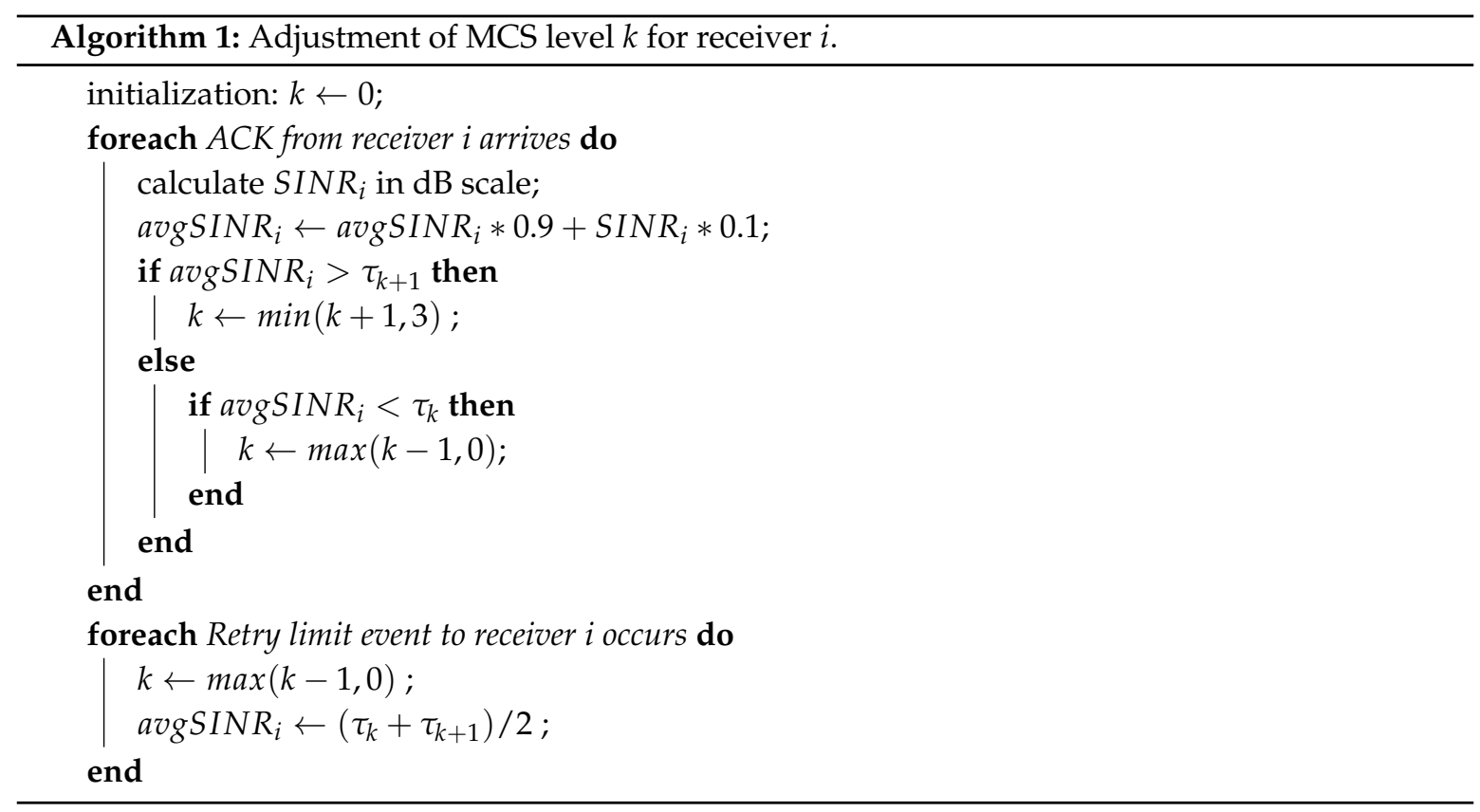

\section{Demonstrations Using Simulations}

\subsection{Simulation Environments}

For demonstrating and evaluating the proposed MCS level adaptation algorithm, we consider IEEE 802.11a wireless networks composed of access points (APs) and wireless devices (WDs) in a given area. All the nodes in this paper use $100 \mathrm{~mW}$ for transmit power and the wireless devices are associated with (i.e., connected to) their serving access points chosen for the strongest received signal strength using Equation (1) before starting the simulations. For the radio transmission model in Equation (1), we modify the two-ray ground model [31] in ns-2 to use the fixed path loss exponent (i.e., $\alpha=4$ ).

In this paper, we consider only downlink traffic from APs to their associated wireless devices (i.e., one-hop transmissions). Thus, there is no direct data traffic between wireless devices. For fixed and bidirectional routing paths between APs and their associated wireless devices, we use the NO Ad Hoc Routing Agent (NOAH) [32]. All participating nodes use the distributed coordination function (DCF) [8] to avoid collisions. When more than one wireless device are associated with the same access point, the wireless devices share the resource of their serving access point.

A packet is recognized as received (or arrived) at a node when the received signal strength of the packet is higher than a power monitoring threshold, which is set to $-129 \mathrm{dBW}$ in this paper. Thus, the power monitoring range (i.e., packet delivery range) is $\sim 1417 \mathrm{~m}$ from each $\mathrm{AP}$ of $100 \mathrm{~mW}$ transmit power. However, packets are recognized as received successfully only if the SINRs of the packets remain higher than the minimum SINR requirements of their MCS levels during the time they are being received (Note that the SINR of a packet can change during the time it is being received owing to the change in cumulative power).

Access points are assumed to be saturated during simulations (i.e., APs are continuously backlogged by a constant-bit-rate (CBR) traffic of $30 \mathrm{Mbps}$ to each wireless device associated with them). The packet size of the CBR traffic is fixed at 1000 bytes and output queue sizes of APs and wireless devices are set to 50 packets. The RTS/CTS scheme is not used and the (physical) carrier sensing range is set to $\sim 532 \mathrm{~m}$ with the $100 \mathrm{~mW}$ transmit power. All other parameters for simulations remain at the default values suggested for IEEE 802.11a wireless networks in [29] unless stated otherwise. 


\subsection{A Single User in a Single Cell}

To demonstrate how the proposed MCS level adaptation algorithm works, we first use the simple topology shown in Figure 2. The figure shows a simulation environment in which there are only one wireless device (WD1) and one access point (AP0) in an area of $2000 \times 2000 \mathrm{~m}$.

In the figure, the solid arrow line from AP0 to WD1 represents the CBR data flow from AP0 to WD1. In addition, as shown with the dashed arrow line in the figure, WD1 moves from its initial location of $(681,240)$ to its final location of $(1800,1800)$ during $10 \mathrm{~s}$ of simulation time. AP0 is located at about $(1334,1001)$.

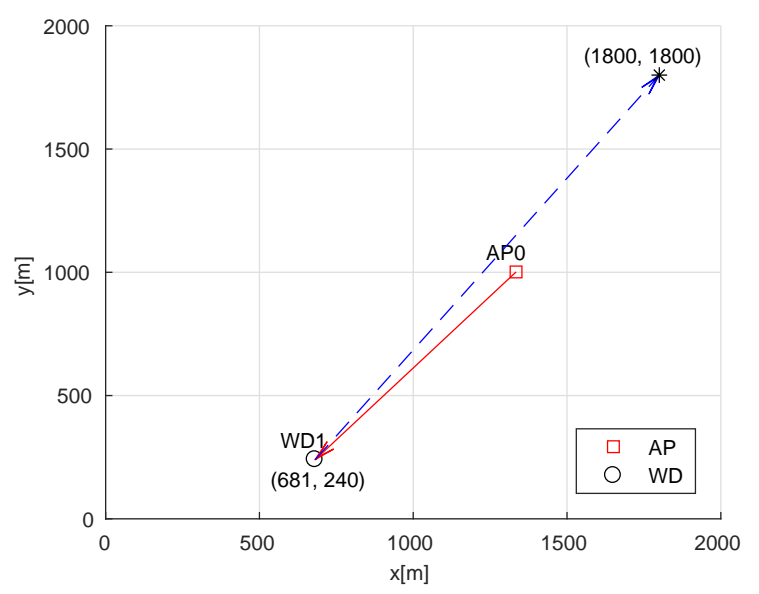

Figure 2. WD1 moves passing its serving access point (AP0) in an area of $2000 \times 2000 \mathrm{~m}$.

In Figure 3a, we show the changes of SINRs measured at AP0 using received ACK frames from WD1 during $10 \mathrm{~s}$ of simulation time. In the figure, we also show the minimum SINR requirements of the four MCS levels in Table 1 with dashed lines.

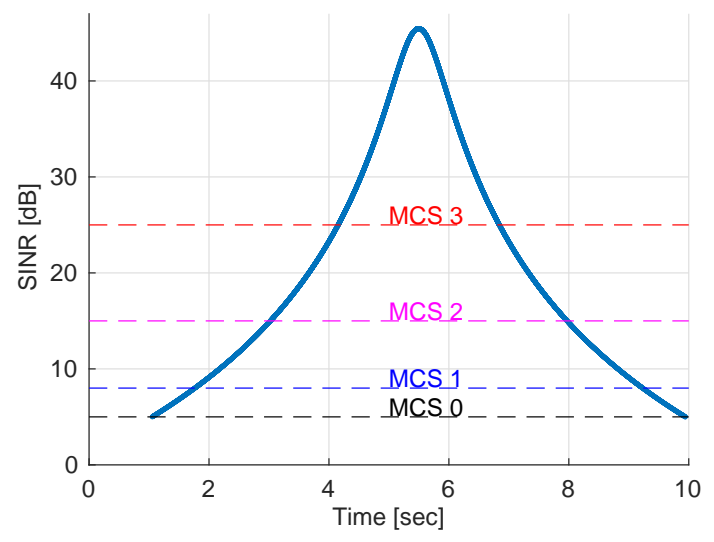

(a)

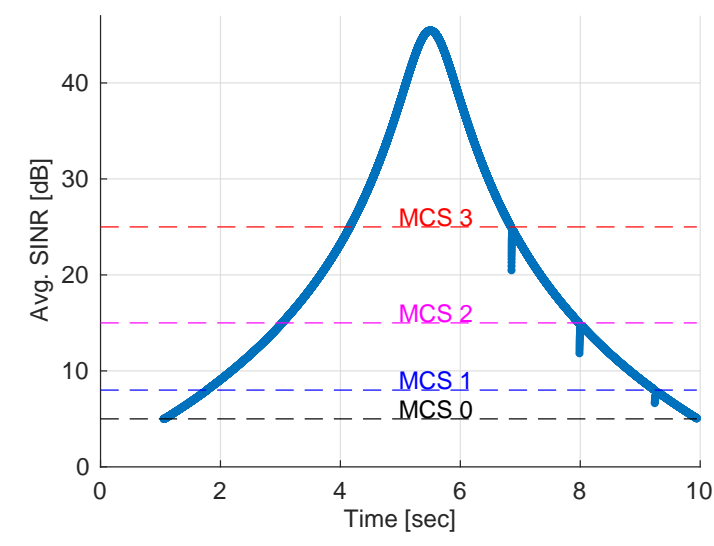

(b)

Figure 3. (a) changes of SINRs measured at AP0 for WD1; (b) changes of the moving averaged SINRs used at AP0 for WD1.

The graph in Figure 3a demonstrates that the sender (i.e., AP0) measures the changes of SINRs of the ACK frames from the receiver (i.e., WD1) as expected. However, note that the results in Figure $3 a$ are the same as the changes of signal-to-noise ratio (SNR) since there are no interfering nodes in this scenario. Also notice that, before $\sim 1 \mathrm{~s}$ of simulation time, the SINRs are not available at AP0 for WD1 since no ACK frames from WD1 arrive at AP0 during this time. This is because the CBR packets from 
$\mathrm{AP0}$ arriving at WD1 during this time are not received successfully since they have lower SINRs than the minimum SINR requirement of MCS level 0 owing to the distance between AP0 and WD1.

In Figure 3b, we show the changes of the moving averaged SINRs (i.e., avgSINRs) used at AP0 for WD1 while WD1 is moving during the $10 \mathrm{~s}$. By comparing Figure 3a with Figure 3b, we can see that the two graphs are similar to each other, except for a few dips when the moving averaged SINRs pass below the minimum SINR requirements of the MCS levels. This occurs because we set avgSINR to the middle value of the new minimum SINR requirement and the previous minimum SINR requirement when a retry limit event is detected. Note that $\operatorname{avgSINR}$ is used not only to represent channel status but also to control the MCS levels of data packets in the proposed algorithm.

We show the changes of the MCS level in AP0 for WD1 and the changes of the achieved throughputs by WD1 during $10 \mathrm{~s}$ of simulation time in Figure $4 \mathrm{a}, \mathrm{b}$, respectively. By comparing the changes of the moving averaged SINRs shown in Figure 3b with the changes of the MCS level in Figure 4a, we can see that AP0 controls the MCS level for WD1 according to the proposed algorithm. From the changes of the achieved throughputs by WD1 shown in Figure 4b, we can see that WD1 achieves a throughput corresponding to each MCS level (The achieved throughputs are lower than the transmission speeds in Table 1 because we are measuring application level throughput and the CSMA/CA mechanism consumes bandwidth.) set by the sender AP0. Also notice that, before $\sim 1 \mathrm{~s}$ of simulation time, WD1 does not achieve any throughput since WD1 is too far from its serving access point AP0 even for the lowest MCS level (i.e., 0) used during the time.

The simulation results in Figures 3 and 4 clearly demonstrate that the proposed algorithm works as expected although it is used in a no-interference environment.

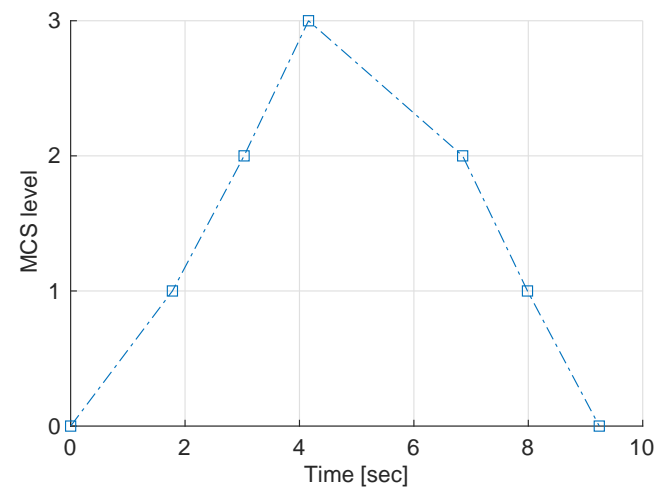

(a)

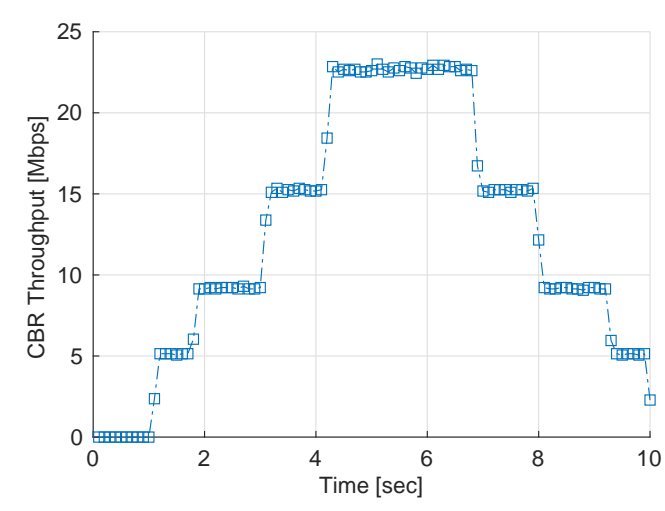

(b)

Figure 4. (a) changes of the MCS level in AP0 for WD1; (b) changes of the achieved throughputs obtained by WD1.

\subsection{Multiple Users in Multiple Cells}

To demonstrate the behavior of the proposed algorithm in an environment with multiple cells (i.e., multiple APs) and multiple users, in Figure 5, we show a Voronoi diagram [33] where 10 APs and 10 wireless devices are located in an area of $2000 \times 2000 \mathrm{~m}$. The APs and the wireless devices in the figure are randomly distributed in the area using two independent homogeneous PPPs of the same density. The solid arrow lines in the figure show connectivities between APs and wireless devices. The lines also represent the CBR traffic flows from APs to their associated wireless devices.

In the figure, notice that some APs (e.g., AP5, AP6, and AP9) do not have any wireless devices associated with them. However, for demonstration purposes, we deliberately change the serving AP for WD10 from AP8 to AP0 and change that for WD13 from AP0 to AP2. As shown with the dashed arrow line in the figure, WD10 moves from its initial location of $(681,240)$ to its final location of $(1800,1800)$ during $10 \mathrm{~s}$ of simulation time. AP0 is located at the same position as in Figure 2. 
In Figure 6a,b, we show the changes of the received signal strengths and SNRs of ACK frames from WD10 arrived at AP0, respectively, during $10 \mathrm{~s}$ of simulation time. The received signal strengths of packets are calculated using Equation (1) by AP0 for every received ACK frame from WD10. The SNRs of ACK frames are calculated using Equation (2) by ignoring interference (i.e., the indicator function is set to 0) when ACK frames from WD10 are received (Note that the SNRs can be obtained by simply deducting the thermal noise power (i.e., $-126 \mathrm{dBW}$ ) from the received signal strengths). In Figure $6 \mathrm{~b}$, we also show the minimum SINR requirements of the four MCS levels in Table 1 with dashed lines (Note that the minimum SINR requirements are the same as the minimum SNR requirements if no interference is assumed).

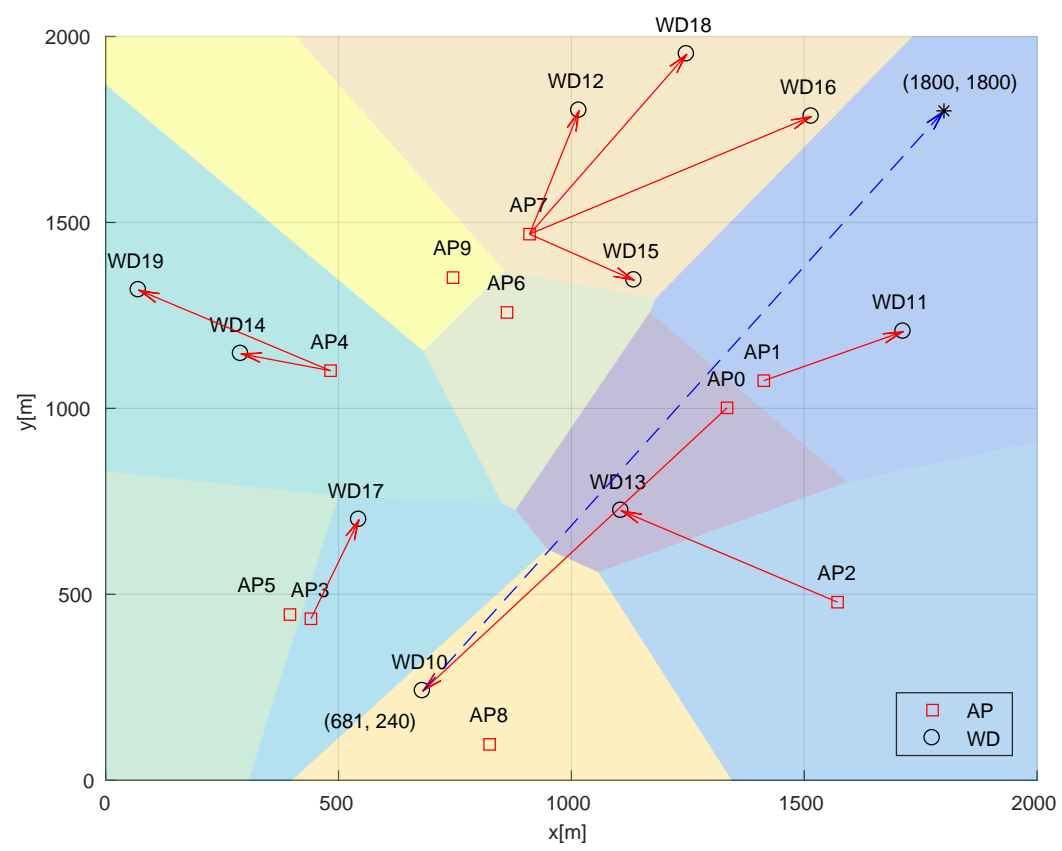

Figure 5. Voronoi diagram of multiple cells and multiple users.

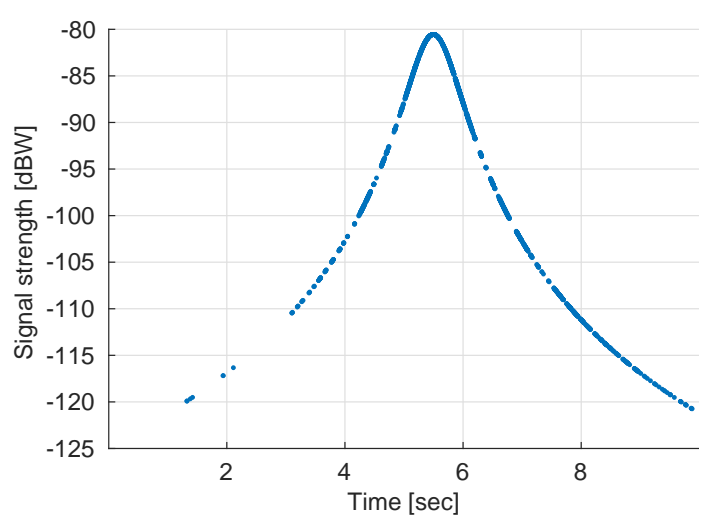

(a)

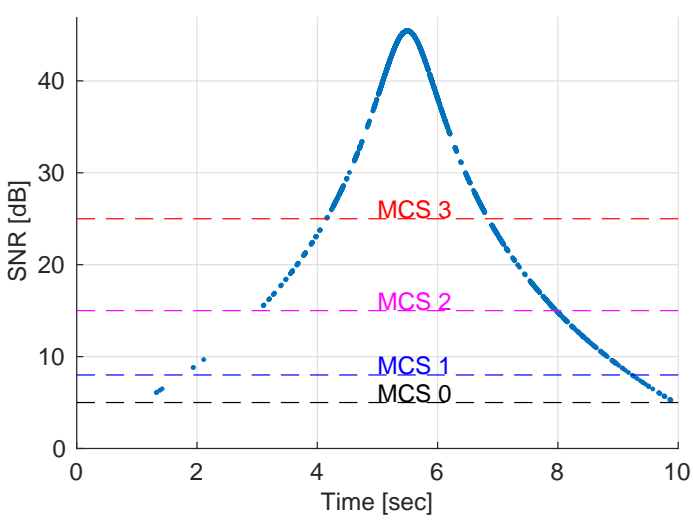

(b)

Figure 6. (a) changes of received signal strengths of ACK frames; (b) changes of SNRs of ACK frames.

By comparing the simulation results in Figure $6 \mathrm{~b}$ with the changes of SINRs of ACK frames shown in Figure 3a, we can see that they are similar to each other. This is because WD10 is moving along the same path and its serving access point (AP0) is located at the same position in both Figures 2 and 5 . The simulation results in Figure 6 also show that AP0 receives less ACK frames from WD10 than in Figure 3a. This is because some of the packets sent by AP0 to WD10 are not received successfully by 
WD10 due to the interference by other nodes in Figure 5 (Note that the proposed algorithm utilizes the absence of ACK frames as shown in Algorithm 1). However, notice that, with the received signal strengths or SNRs of ACK frames, it is hard to capture the intensity of interference in multi-cell, multi-user environments.

In Figure 7a,b, we show the changes of SINRs of ACK frames from WD10 received at AP0 and the moving averaged SINRs calculated by AP0 for WD10 using the SINRs of the ACK frames, respectively, during $10 \mathrm{~s}$ of simulation time. The graphs in Figure 7 show that the SINRs and the moving averaged SINRs of ACK frames experience fluctuations caused by interfering packets from other nodes while WD10 moves. However, from the results, we can see that the moving averaged SINRs fluctuate less than the instantaneous SINRs as expected.

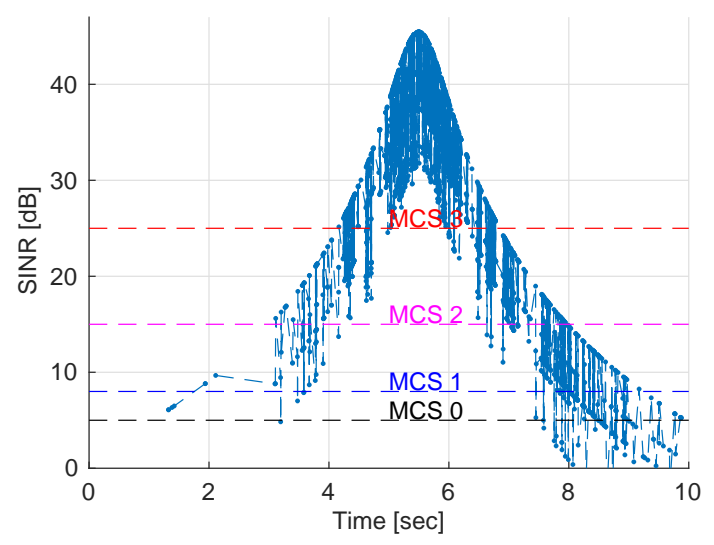

(a)

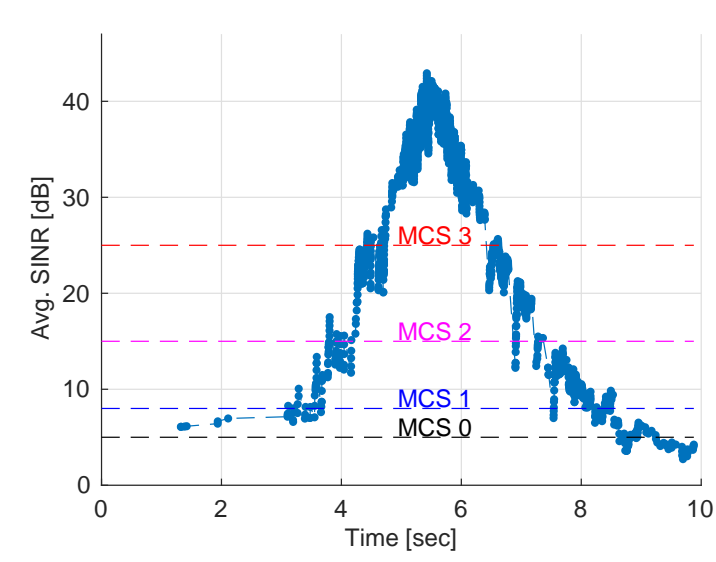

(b)

Figure 7. (a) changes of SINR measured at AP0 for WD10; (b) changes of the moving averaged SINR used at AP0 for WD10.

We also show the changes of the MCS level in AP0 for WD10 and the changes of the achieved throughputs by WD10 during $10 \mathrm{~s}$ of simulation time in Figure 8a,b, respectively. By comparing the graphs of the moving averaged SINRs in Figure $7 \mathrm{~b}$ with the changes of the MCS level in Figure 8a, we can see that the MCS level at AP0 for WD10 reflects the changes of the moving averaged SINRs for WD10.

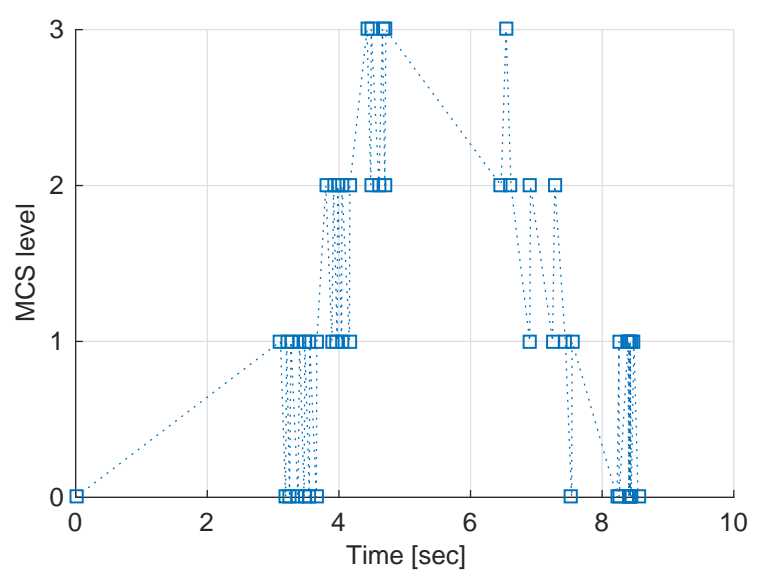

(a)

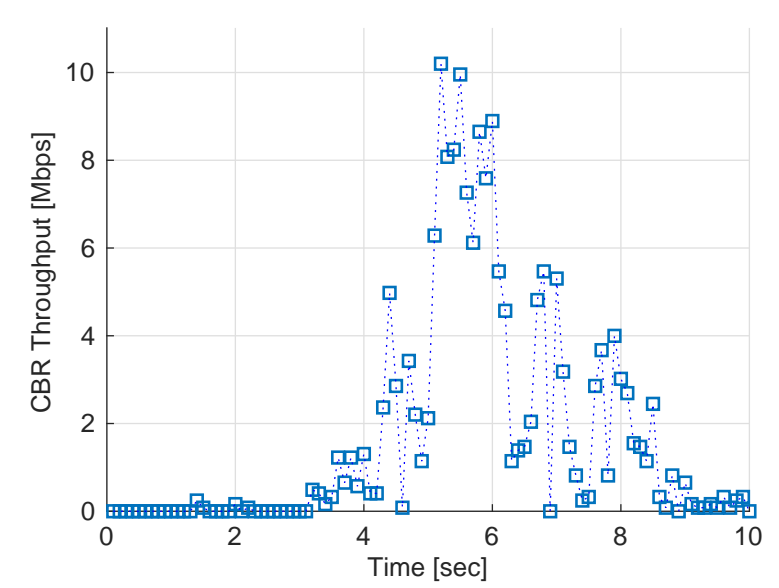

(b)

Figure 8. (a) changes of the MCS level in AP0 for WD10; (b) changes of the achieved throughputs obtained by WD10. 
The simulation results of the achieved throughputs by WD10 in Figure $8 b$ show that WD10 achieves less than the corresponding throughput of each MCS level. This is because AP0 (along with WD10) shares network resources with other nodes in this case according to the CSMA/CA mechanism. From the simulation results in Figures 7 and 8, we can see that the proposed algorithm works as expected in this multi-cell, multi-user environment.

\section{Performance Evaluation}

To evaluate the performance of the proposed algorithm, we conducted simulations with various combinations of the numbers of APs and users using different MCS levels and the proposed algorithm. In all the simulations, APs and users (i.e., wireless devices) are randomly distributed using two independent homogeneous PPPs in a given area. All participating APs start sending CBR traffic of $30 \mathrm{Mbps}$ to each wireless node associated with them when the simulations start. All simulations run for $2 \mathrm{~s}$ of simulation time and all simulation results are averaged values from 10 repetitions with different seed values for randomness unless stated otherwise.

\subsection{The Same Number of Access Points and Users}

In this section, we first show the simulation results when both the number of APs and the number of wireless devices increase from 2 to 60 (i.e., 2, 4, 6, 10, 20, 30, 40, and 60) in a circular area with a radius of $1000 \mathrm{~m}$. In Figure 9, we show the total (i.e., aggregated) throughputs achieved by all wireless devices when a fixed MCS level is used for communications of all participants in different PPP densities of APs for $2 \mathrm{~s}$. The total throughputs are averaged values from 10 repetitions of each scenario. From the simulation results in Figure 9, we can see a trend of higher total achieved throughputs with higher MCS levels and higher densities of APs (and wireless devices).

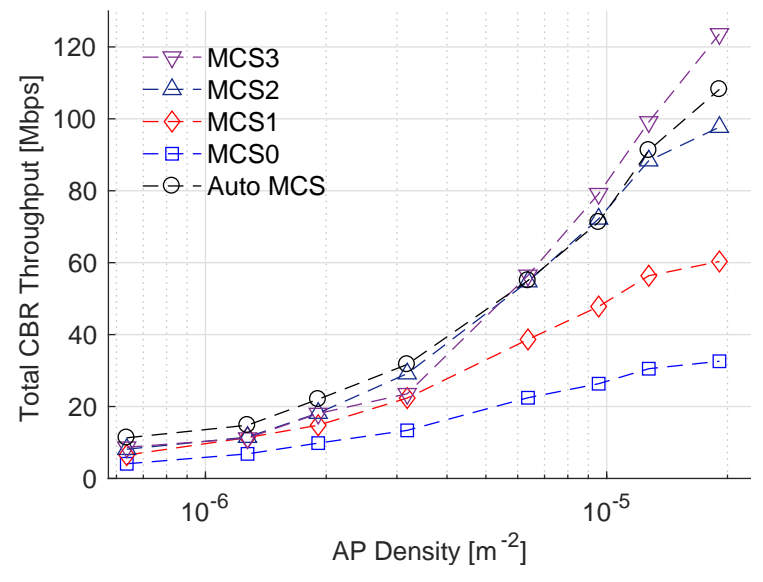

Figure 9. Total achieved throughputs.

In Figure 9, we also show total throughputs achieved by all wireless devices when all APs use the proposed automatic MCS level adaption algorithm. The simulation results in Figure 9 show that APs using the proposed algorithm utilize the resources of the simulated IEEE 802.11a wireless networks almost as effectively as the highest MCS level in Table 1 (i.e., 3), although the APs do not always use the highest MCS level.

In Figure 10, we show the ratios (in percent) of the number of discarded packets by retry limit events to the number of packets sent by APs during $2 \mathrm{~s}$ of simulation time. In CSMA/CA wireless networks, if a sender does not receive an ACK frame corresponding to the transmitted packet in a given time, which is decided by the short interframe space (SIFS) [8] and the transmission speeds of MCS levels, it is counted as a timeout. The sender retransmits the timeouted packet until the number of consecutive retransmissions for the packet reaches a limit called the retry limit. When the number 
of retransmissions for a packet reaches the retry limit (i.e., a retry limit event occurs), the packet is discarded by the sender.

The simulation results in Figure 10 show a trend of higher retry limit ratios with higher MCS levels. In Figure 10, we also show the simulation results of the retry limit ratios when all APs use the proposed automatic MCS level adaption algorithm. From the results in the figure, we can see that the proposed algorithm keeps the retry limit ratios comparable to those of the lowest MCS level (i.e., 0) for most AP densities. The simulation results in Figures 9 and 10 clearly show that the proposed algorithm effectively and efficiently utilizes network resources by adapting the MCS levels according to the environments of the IEEE 802.11a wireless networks.

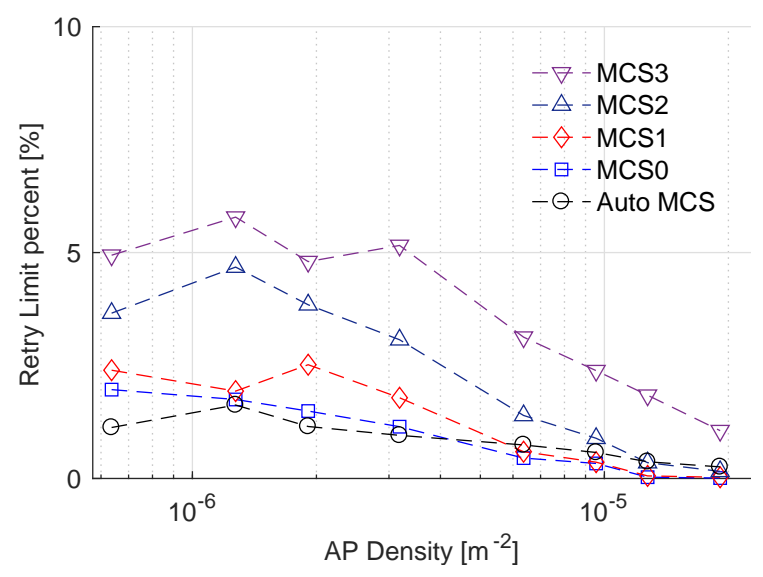

Figure 10. Ratios of retry limits to sent packets.

In Figure 11, we show the average values of the time-averaged MCS levels used by all APs for their associated wireless devices during $2 \mathrm{~s}$ of simulation time with $95 \%$ confidence intervals. The simulation results show that the APs using the proposed algorithm increase their MCS levels when the densities of APs and wireless devices increase. This result implies that the overall SINRs of the wireless networks are increasing as the PPP density of APs increases. This is because the received signal strengths increase as the distances between wireless devices and their serving APs decrease while the CSMA/CA mechanism controls interference among participating nodes.

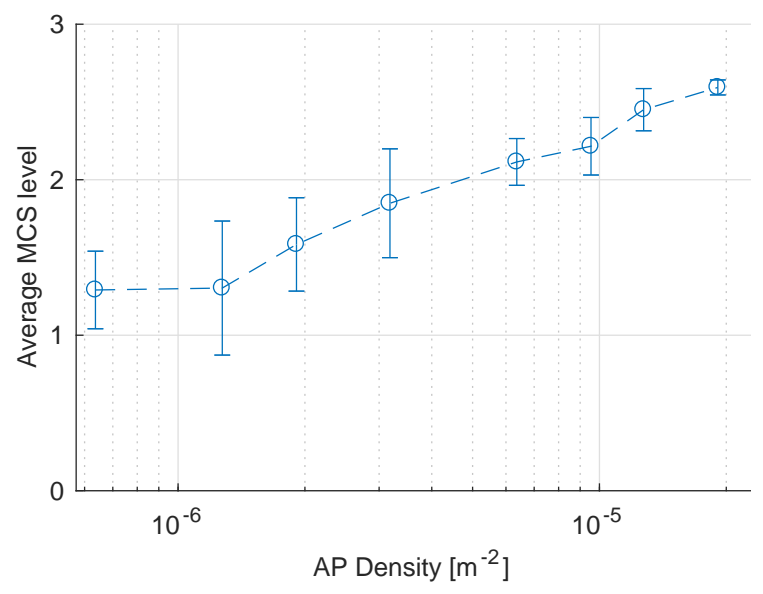

Figure 11. Average MCS levels with 95\% confidence intervals.

In addition, in Figure 12, we show cumulative distributions of user throughputs with different MCS levels when both the number of APs and the number of wireless devices are 60 . The simulation results in the figure show that there is higher dispersion of throughputs when higher MCS levels are 
used for communication. In the figure, the cumulative distributions of user throughputs with the proposed algorithm show less dispersion than that of the highest MCS level (i.e., 3).

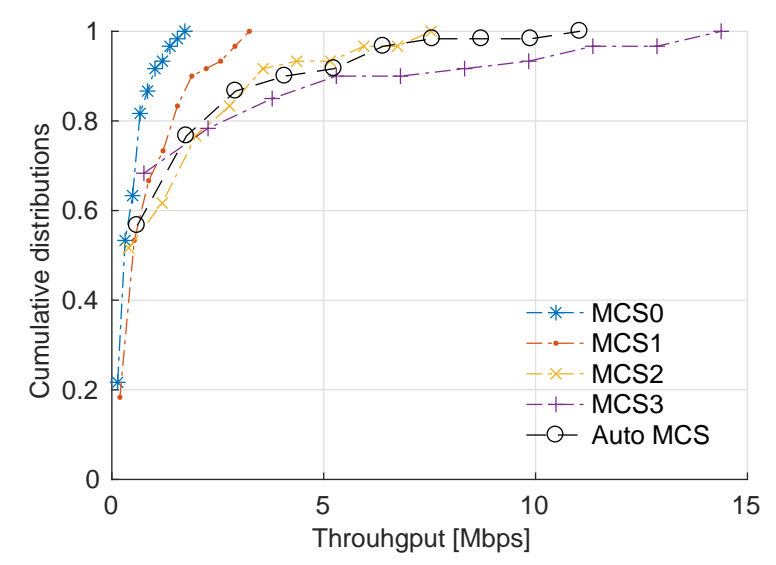

Figure 12. Cumulative distributions of user throughputs.

In Figure 13, we show the fairness among users using the achieved throughput by each user with different MCS levels for different AP densities. To measure fairness among user throughputs, we use the fairness index (FI) from [34] defined as

$$
F I\left(T_{1}, T_{2}, \ldots, T_{n}\right)=\frac{\left(\sum_{i=1}^{n} T_{i}\right)^{2}}{n \sum_{i=1}^{n} T_{i}^{2}}
$$

where $T_{i}$ is the achieved throughput by user $i$. The results in Figure 13 show that users achieve better fairness with the proposed algorithm than with the highest MCS level.

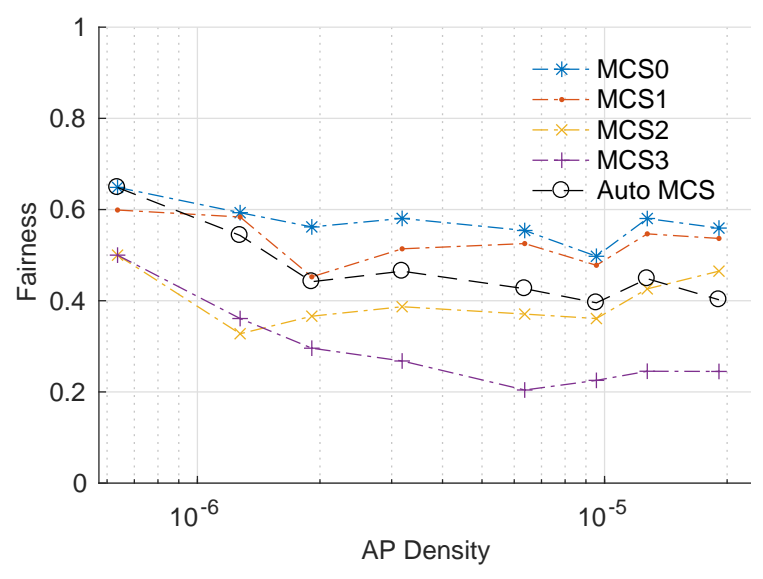

Figure 13. Fairness among user throughputs.

\subsection{Fixed Number of Users}

In this section, we consider a scenario in which an increasing number of APs is introduced to serve a fixed number of wireless devices in CSMA/CA wireless networks. For this, we fix the number of wireless devices at 60 and increase the number of APs from 2 to 60 (i.e., 2, 4, 6, 10, 20, 30, 40, and 60) in a circular area with a radius of $1000 \mathrm{~m}$.

In Figure 14, we show the total throughputs achieved by the 60 wireless devices when different MCS levels are used for different PPP densities of APs for $2 \mathrm{~s}$ of simulation time. From the simulation results in the figure, we can see a pattern of higher total achieved throughputs with higher MCS levels and higher densities of APs, similarly to the results in Figure 9. The figure also shows the total 
throughputs achieved by the 60 wireless devices when all APs use the proposed automatic MCS level adaption algorithm. From the results, we can see that the 60 wireless devices achieve throughputs comparable to those achieved when all APs use the highest MCS level.

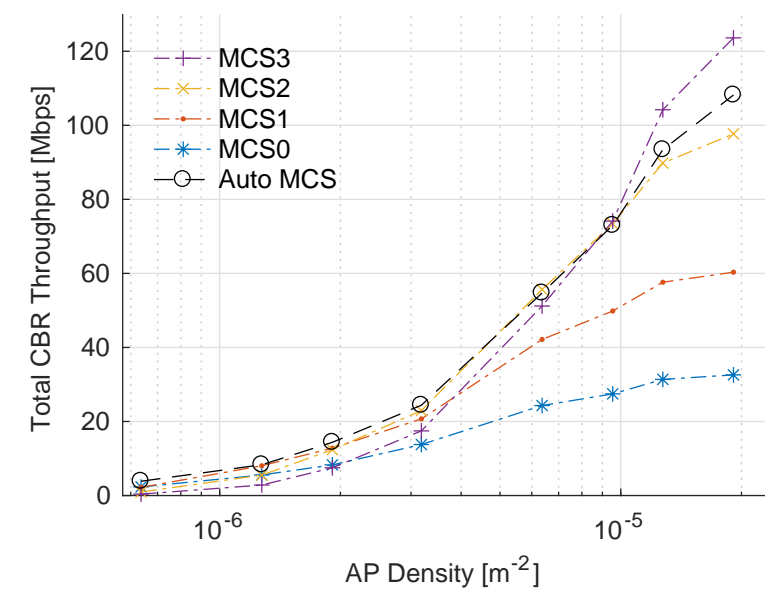

Figure 14. Total throughputs achieved by 60 wireless devices.

In Figure 15, we show the retry limit ratios of all the packets sent by APs for $2 \mathrm{~s}$ of simulation time. Similarly to the results in Figure 10, the simulation results in Figure 15 also show that the proposed algorithm keeps the retry limit ratios comparable to those of the lowest MCS level in most cases. However, the simulation results in Figure 15 show higher retry limits ratios for low densities of APs compared to those in Figure 10. This is partly because many wireless devices are located outside the minimum distances (The minimum distances for the MCS levels can be calculated using the minimum SINR requirements of the MCS levels and the transmit power by assuming no interference.) for their MCS levels but inside the packet delivery ranges (i.e., power monitoring ranges) from their serving APs when only small numbers of APs exist in the relatively large area we simulated.

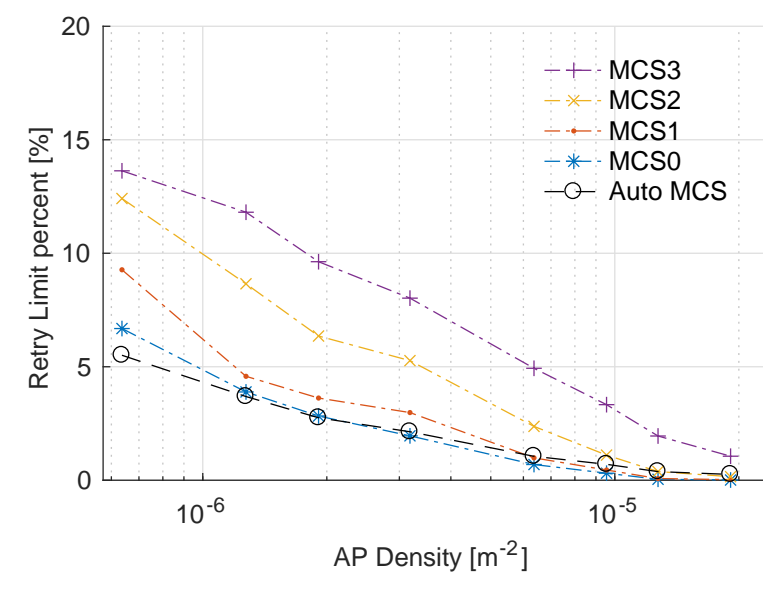

Figure 15. Ratios of retry limits to sent packets.

In Figure 16, we show the throughput fairness among the 60 wireless devices with different MCS levels for different densities of APs. The results show that users achieve better fairness with the proposed algorithm than with the highest MCS level, similarly to the results in Figure 13. 


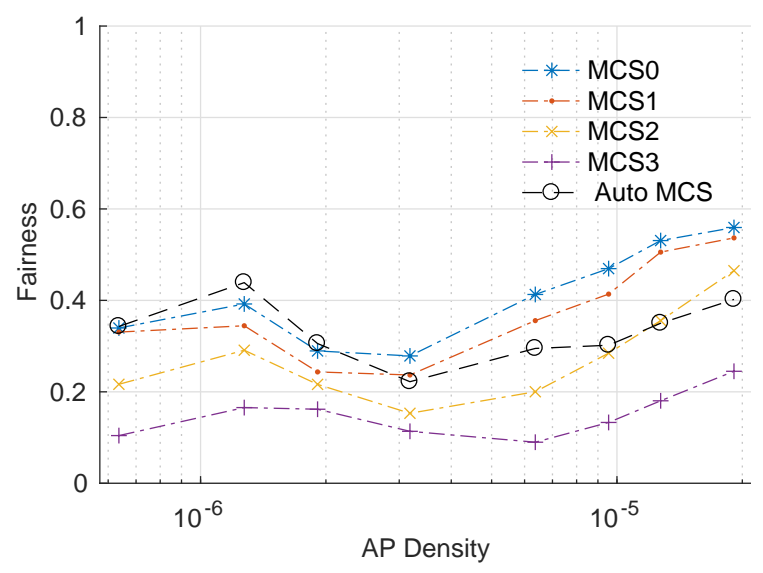

Figure 16. Fairness among user throughputs of 60 wireless devices.

\subsection{Fixed Number of Access Points}

In this section, we consider a scenario in which the numbers of users are increasing while the number of APs is fixed. For this, we fix the number of APs at 30 and increase the number of wireless devices from 2 to 60 (i.e., 2, 4, 6, 10, 20, 30, 40, and 60) in a circular area with a radius of $1000 \mathrm{~m}$.

In Figure 17, we show the total throughputs achieved by the varying numbers of the wireless devices when different MCS levels are used for different PPP densities of users (i.e., wireless devices) for $2 \mathrm{~s}$ of simulation time. The simulation results in the figure show that the total achieved throughputs obtained by users increase when higher MCS levels are used for communication. However, differently from the results in Figures 9 and 14, the results in Figure 17 show that the total achieved throughputs do not increase much after the number of users reaches 20 . This is because, in this scenario, the number of APs is fixed, and the wireless devices associated with the same AP share resources of their serving AP. In Figure 17, we also show the total achieved throughputs by the varying numbers of the wireless devices when all the 30 APs use the proposed automatic MCS level adaption algorithm. From the results, we can see that the proposed algorithm results in total throughputs comparable to those achieved by using high MCS levels such as 2 and 3.

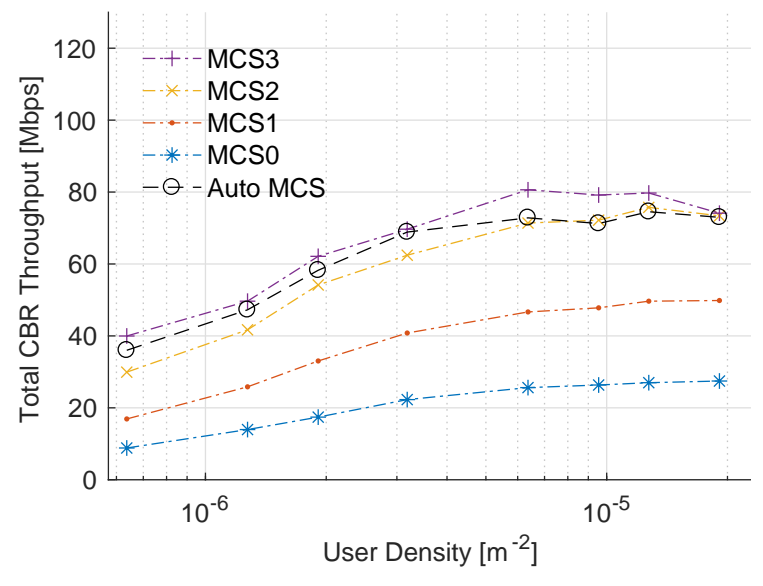

Figure 17. Total achieved throughputs.

In Figure 18, we show the retry limit ratios of the packets sent by the 30 APs for $2 \mathrm{~s}$ of simulation time. The simulation results in the figure show that the proposed algorithm keeps the retry limit ratios lower than those of high MCS levels such as 2 and 3 in most cases. From the figure, we can also see the difference between the retry limit ratio of the highest MCS level and those of other MCS levels becomes larger when more wireless devices are introduced in the area. 


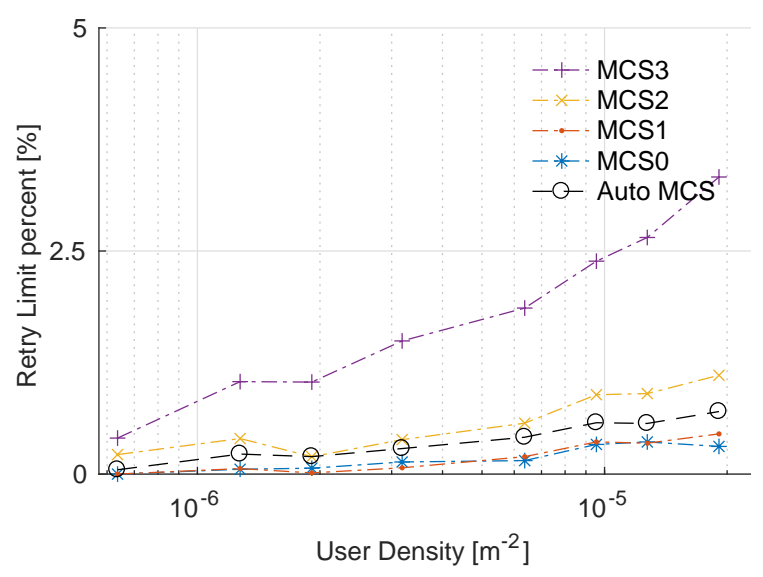

Figure 18. Ratios of retry limits to sent packets.

In Figure 19, we show the throughput fairness among the varying numbers of the wireless devices with different MCS levels when the number of APs is fixed at 30. The results in the figure show that users achieve better fairness with the proposed algorithm than with the highest MCS level, similarly to the results in Figures 13 and 16. However, the results show high fairness for low densities of users compared to the results in Figures 13 and 16. This is partly because, when there are only small numbers of wireless devices in the area we simulated, the APs associated with them are located relatively far from each other because of the PPP density of the APs.

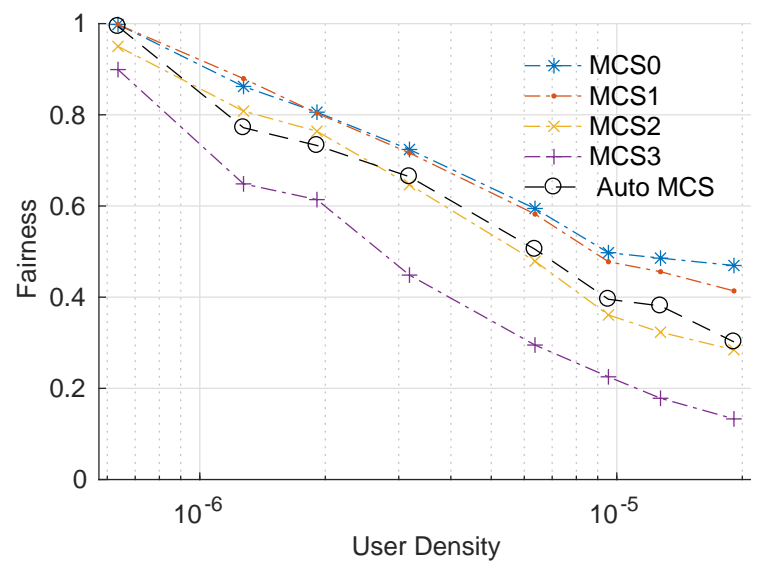

Figure 19. Fairness among user throughputs.

\section{Conclusions}

In this paper, we propose an automatic MCS level adaptation algorithm using SINRs of ACK frames in CSMA/CA wireless networks to accommodate the expected high density of IoT devices in CSMA/CA wireless networks. The proposed algorithm does not require any modification of the existing CSMA/CA mechanism because it is based on the information from normal ACK frames in IEEE 802.11 standards. Our simulation results show that the proposed algorithm utilizes resources of IEEE 802.11a wireless networks almost as effectively as the highest MCS level of a given MCS scheme for various combinations of PPP densities of access points and wireless devices.

In addition, we show through simulations that the proposed algorithm results in low retry limit ratios in sent packets compared to those of the highest MCS level. Furthermore, the fairness among per user throughputs also exhibits better results than those of the highest MCS level. Since the proposed algorithm works in a purely distributed manner and is less aggressive than the highest MCS level, we expect other performance improvements to be further revealed in the near future. 
Acknowledgments: This work was supported by the Hongik University new faculty research support fund.

Conflicts of Interest: The author declares no conflicts of interest.

\section{References}

1. Atzori, L.; Iera, A.; Morabito, G. The Internet of Things: A survey. Comput. Netw. 2010, 54, 2787-2805.

2. Kang, W.M.; Moon, S.Y.; Park, J.H. An enhanced security framework for home appliances in smart home. Hum.-Centric Comput. Inf. Sci. 2017, 7. doi:10.1186/s13673-017-0087-4.

3. Sharma, P.; Moon, S.Y.; Park, J.H. Block-VN: A distributed blockchain based vehicular network architecture in smart City. J. Inf. Proc. Syst. 2017, 13, 184-195.

4. Lee, J.K.; Jeong, Y.S.; Park, J.H. s-ITSF: A service based intelligent transportation system framework for smart accident management. Hum.-Centric Comp. Inf. Sci. 2015, 5, 34.

5. Maity, S.; Park, J.H. Powering IoT devices: A novel design and analysis technique. J. Converg. 2016, 7, 16071001 .

6. Stankovic, J.G. Research directions for Internet of Things. IEEE Internet Things J. 2014, 1, 3-9.

7. Ratasuk, R.; Prasad, A.; Li, Z.; Ghosh, A.; Uusitalo, M.A. Recent advancements in M2M communications in 4G networks and evolution toward 5G. In Proceedings of the 2015 18th International Conference on Intelligence in Next Generation Networks (ICIN), Paris, France, 17-19 Feburary 2015.

8. IEEE Computer Soceity. IEEE Std 802.11-2016 Part 11: Wireless LAN Medium Access Control (MAC) and Physical Layer (PHY) Specifications; IEEE Computer Soceity: Washington, DC, USA, 2016.

9. IEEE Computer Society. IEEE Std 802.15.4-2015: IEEE Standard for Low-Rate Wireless Networks; IEEE Computer Soceity: Washington, DC, USA, 2015.

10. Weber, S.; Andrews, J.G.; Jindal, N. An overview of the transmission capacity of wireless networks. IEEE Trans. Commun. 2010, 58, 3593-3604.

11. Chiu, S.; Stoyan, D.; Kendall, W.S.; Mecke, J. Stochastic Geometry and Its Applications, 3rd ed.; Wiley: New York, NY, USA, 2013.

12. Zhang, J.; Tan, K.; Zhao, J.; Wu, H.; Zhang, Y. A practical SNR-guided rate adaptation. In Proceedings of the IEEE INFOCOM 2008 27th Conference on Computer Communications, Phoenix, AZ, USA, 13-18 April 2008.

13. The Network Simulator-ns-2. Available online: http://www.isi.edu/nsnam/ns (accessed on 1 September 2017).

14. IEEE Computer Society. IEEE Std 802.11a-1999 Part 11: Wireless LAN Medium Access Control (MAC) and Physical Layer (PHY): High-Speed Physical Layer in the 5 GHZ Band; IEEE Computer Soceity: Washington, DC, USA, 1999.

15. Kim, J.; Kim, S.; Choi, S.; Qiao, D. CARA: Collision-aware rate adaptation for IEEE 802.11 WLANs. In Proceedings of the 25th IEEE International Conference on Computer Communications (INFOCOM 2006), Barcelona, Spain, 23-29 April 2006.

16. Chen, C.; Luo, H.; Seo, E.; Vaidya, N.H.; Wang, X. Rate-adaptive framing for interfered wireless networks. In Proceedings of the 26th IEEE International Conference on Computer Communications (IEEE INFOCOM 2007), Barcelona, Spain, 6-12 May 2007.

17. Vutukuru, M.; Balakrishnan, H.; Jamieson, K. Cross-layer wireless bit rate adaptation. In Proceedings of the ACM SIGCOMM 2009 conference on Data communication (SIGCOMM '09), Barcelona, Spain, 16-21 August 2009.

18. Lee, O.; Kim, J.; Lim, J.; Choi, S. SIRA: SNR-aware intra-frame rate adaptation tested traces. IEEE Commun. Lett. 2015, 19, 90-93.

19. Kim, W.; Park, J.-S. Cross-layer scheduling for multi-users in cognitive multi-radio mesh networks. Wirel. Commun. Mobile Comput. 2014, 14, 1034-1044.

20. Andrews, J.G.; Baccelli, F.; Ganti, R.K. A tractable approach to coverage and rate in cellular networks. IEEE Trans. Commun. 2011, 59, 3122-3134.

21. Weber, S.; Yang, X.; Andrews, J.G.; Veciana, G. Transmission capacity of wireless ad hoc networks with outage constraints. IEEE Trans. Inf. Theory 2005, 51, 13-17.

22. Roberts, L.G. ALOHA packet system with and without slots and capture. Comput. Commun. Rev. 1975, 5, 28-42. 
23. Nguyen, H.Q.; Baccelli, F.; Kofman, D. A stochastic geometry analysis of dense IEEE 802.11 networks. In Proceedings of the 26th IEEE International Conference on Computer Communications (IEEE INFOCOM 2007), Barcelona, Spain, 6-12 May 2007.

24. Haenggi, M., Mean interference in hard-core wireless networks. IEEE Commun. Lett. 2011, 15, 792-794.

25. Alfano, G.; Garetto, M.; Leonardi, E. New directions into the stochastic geometry analysis of dense CSMA metwork. IEEE Trans. Mob. Comput. 2014 13, 324-336.

26. Chen, Q.; Schmidt-Eisenlohr, F.; Jiang, D. Overhaul of IEEE 802.11 modeling and simulation in NS-2. In Proceedings of the 10th ACM Symposium on Modeling, Analysis, and Simulation of Wireless and Mobile Systems, Chania, Crete Island, Greece, 22-26 October 2007, pp. 159-168.

27. Schmidt-Eisenlohr, F. Interference in Vehicle-to-Vehicle Communication Networks-Analysis, Modeling, Simulation and Assessment; KIT Scientific Publishing: Karlsruhe, Germany, 2010.

28. ElSawy, H.; Hossain, E.; Haenggi, M. Stochastic geometry for modeling, analysis, and design of multi-tier and cognitive cellular wireless networks: A Survey. IEEE Commun. Surv. Tutor. 2013, 15, 996-1019.

29. Chen, Q.; Schmidt-Eisenlohr, F.; Jiang, D.; Torrent-Moreno, M.; Delgrossi, L.; Hartenstein, H. Overhaul of IEEE 802.11 Modeling and Simulation in NS-2 (802.11Ext). Available online: https://dsn.tm.kit.edu/ medien/downloads_old/Documentation-NS-2-80211Ext-2008-02-22.pdf (accessed on 1 September 2017).

30. Postel, J. Transmission Control Protocol (RFC 793), 1981. Available online: http:/ /www.ietf.org/rfc/rfc793.txt (accessed on 1 September 2017).

31. Two-ray Ground Reflection Model in NS Version 2 Manual. Available online: http://www.isi.edu/nsnam/ ns/doc/node218.html (accessed on 1 September 2017).

32. NO Ad-hoc Routing Agent. Available online: http://icapeople.epfl.ch/widmer/uwb/ns-2/noah (accessed on 1 September 2017).

33. Okabe, A.; Boots, B.; Sugihara K.; Chiu, S.N. Spatial Tessellations: Concepts and Applications of Voronoi Diagrams; Wiley: New York, NY, USA, 2000.

34. Chiu, D.; Jain, R. Analysis of the increase and decrease algorithm for congestion avoidance in computer networks. J. Comput. Netw. ISDN 1989, 17, 1-14.

(C) 2017 by the author. Licensee MDPI, Basel, Switzerland. This article is an open access article distributed under the terms and conditions of the Creative Commons Attribution (CC BY) license (http:/ / creativecommons.org/licenses/by/4.0/). 